\title{
Toxoplasmosis, a rare case report that led to a successful pregnancy.
}

\author{
THOMAS NTOUNIS ${ }^{1}$, Sofoklis Stavros ${ }^{1}$, Antonios Koutras ${ }^{1}$, Alexandros Katrachouras ${ }^{2}$, \\ Dimitrios Lentzaris ${ }^{2}$, Ekaterini Domali ${ }^{1}$, Kyriakos Konis ${ }^{2}$, and Peter Drakakis ${ }^{1}$ \\ ${ }^{1}$ National and Kapodistrian University of Athens \\ ${ }^{2}$ General Hospital of Arta
}

April 17, 2021

\begin{abstract}
A 19-year-old pregnant patient was diagnosed with primary toxoplasmosis infection. The patient was successfully treated with spiramycin and finally delivered normally a healthy boy. Serological tests for detection of anti-toxoplasma antibodies along with ultrasonography findings are the gold standard for the diagnosis. Spiramycin was used for embryo protection.
\end{abstract}

\section{Introduction}

Toxoplasmosis is one of the most common infections of humans worldwide (1). T. gondii (toxoplasma gondii) is an obligate intracellular parasite. Although replication of the pathogen takes part in the cat's intestine, there are several routes of transmission in humans with the main one being the oral route. No transmission during breastfeeding or direct human-human transmission has been recorded $(1,2)$. On the other hand, vertical transmission from mother to fetus via the placenta occurs and the severity of its manifestations is inversely related to gestational age accompanied by several clinical and prenatal ultrasonographic findings. Primary Maternal infection on the 1st and 2nd trimester can result in severe congenital toxoplasmosis, with the 1st trimester maternal infection leading to the most severe manifestations (3). Congenital toxoplasmosis has a wide range of clinical manifestations ranging from mild to severe with chorioretinitis, hydrocephalus and intracranial calcifications constitute the so-called classic triad (1). Therefore, early detection and medical intervention is necessary either to prevent embryo infection or treat embryos when infection is confirmed or suspected. Herein we report one of the few cases based on the literature of toxoplasmosis led to a successful pregnancy in a 19-year-old girl. Not only her young age but also her successful pregnancy as well contributes to its interest for publication.

\section{Case history/examination}

A 19-year-old female patient, was presented at the antenatal outpatient section of our hospital for pregnancy follow-up. This was her first pregnancy (G1P0) and she had no other past medical history. She was currently on the 12 th week of gestation during which on routine tests, was found positive for toxoplasmosis. The patient did not report coming in contact with cats or consuming not properly prepared foods.

Differential diagnosis, investigations and treatment

Her initial serologic tests immediately after the first visit were: IgG $249 \mathrm{IU} / \mathrm{ml}$ (normal range 0-3 IU/ml) and an index of IgM 1.640 (normal range 0-0.6). All the rest antenatal tests were normal. The tests were repeated after 2 weeks and the new laboratory results were IgG $723 \mathrm{IU} / \mathrm{ml}$ and an index of IgM 1.390. During this time first trimester ultrasound and associated blood tests were performed (NT scan and PAPP-A, free b-hCG), which were found within normal range (Figure 1). 
Five days later new exams were requested, with the new results being: IgG: $203 \mathrm{IU} / \mathrm{ml}$, an IgM index of 1.38 and an IgG avidity of 49.0 (with a reference value of $<50$ indicating low avidity). For confirmation of our results, after a week a new sample was sent to the Hellenic Pasteur Institute with ELISA results being $\mathrm{IgG}>200 \mathrm{IU} / \mathrm{ml}$ (reference value of positive results $>11$, an index of IgM 2.61 (reference value of positive results $>1.1$ ) and IgG avidity $23 \%$ (with a reference value $<40 \%$ ) indicating a recent primary toxoplasmosis infection possibly within the last 3 months.

In view of these findings on the 18th week of pregnancy and 2 weeks after the last blood tests performed, an amniocentesis was requested in order to examine whether the fetus was affected. PCR-DNA test of the amniotic fluid was negative. Although there was no evidence of vertical transmission, the infection was confirmed and the patient received treatment with spiramycin 1 gr three times a day (t.i.d.).

Additional serologic test of the toxoplasma gondii IgG and $\operatorname{IgM}$ values was performed on the 20th week of gestation found to be: IgG $149 \mathrm{IU} / \mathrm{ml}$ (with reference value of positive result $>3$ ) and IgM index 0.4 (reference value of negative result $<0.5$ )

Second trimester ultrasound was performed on the 31st week of pregnancy were no pathologic anatomical signs found.

\section{Outcome and Follow-up}

The patient on the 39th week of pregnancy was admitted to the hospital with spontaneous labour onset and finally delivered normally.

The newborn was a healthy boy with a birth weight of $3630 \mathrm{gr}$. Serological tests of the child (blood sampling from umbilical cord) was: IgG $480 \mathrm{IU} / \mathrm{ml}$ (considered of maternal origin because newborn also cannot produce IgG) and IgM negative. Tests were repeated one month later with the same results. Soon after birth, a brain ultrasound was performed to the child, which revealed no structural anatomical abnormalities.

\section{Discussion}

\section{Pathogenesis}

Toxoplasma gondii is an obligate intracellular protozoan existing in three forms: the oocyst, the tachyzoite and the bradyzoite. Replication of the parasite takes place in the intestine of the cat family Felidae, as a result the production of oocysts. Millions of oocysts are shed into the feces of cats. After sporulation, oocysts containing sporozoites are infective when ingested by mammals, including humans. At this point the tachyzoite stage starts. The rapidly dividing form of the parasite is represented by the stage of tachyzoites. Active penetration is the way that the parasite contaminates all the nucleated cells. After that, a continuous proliferation leads to host cells destruction and tachyzoites invade and damage adjacent cells (2). Furthermore, they are disseminated via bloodstream and infect many tissues. This situation causes a strong inflammatory response, tissue damage and, therefore clinical manifestations. The host's immune response can put pressure and limit the growth of tachyzoites, and so they are transformed into bradyzoites, which they form tissue cysts (in the brain and skeletal and heart muscles). Bradyzoites stay inside cysts for the entire life of the host and although they are morphologically identical to tachyzoites, multiply slowly. In immunocompromised patients (patients with AIDS, organ transplantation, cancer, or taking immunosuppressants), bradyzoites can be released from cysts and transform back into tachyzoites (1). In addition, tissue cysts are considered to be infective stages for intermediate hosts (2).

\section{Epidemiology}

Toxoplasmosis is one of the most common infections of humans worldwide. The rate of infection rises with age and does not vary between sexes (1). Furthermore, infection is more common at warm climates and at lower altitudes than in cold climates and mountainous regions (2).

\section{Transmission}


There are several routes of transmission but the oral route is the major source of infection, so it's results from either ingestion or handling of undercooked or raw meat (mainly lamb and pork) containing tissue cysts (bradyzoites) or water or food containing oocysts excreted in the feces of infected cats (2). Transmission of Toxoplasma gondii by organ transplantation from a seropositive donor to a seronegative recipient is a potential cause of human being infection (1). Toxoplasma gondii can also be transmitted via blood, thus laboratory personnel are under a great danger by contact contaminated needles (2). In some cases, the specific route of transmission is not clearly defined. Transmission during breastfeeding or direct human-tohuman transmission other than vertical-transplacentally (mother to fetus) has not been recorded $(1,2)$.

\section{Congenital transmission}

Toxoplasma gondii via maternal bloodstream infects the placenta and then enters the fetal circulation. Maternal infection before gestation poses little or no risk to the fetus except than women who become infected just a few months (at the most three) before conception (2). Transmission and severity of disease are determined by the gestational age of which the mother became infected and they are inversely related. So, maternal infection during the first and the second trimester may result in severe congenital toxoplasmosis, abortion, death of the fetus in uterus, perinatal death or stillbirth despite the fact that during those trimesters the risk of congenital transmission is reduced (less than $15 \%$ at 13 weeks of gestation) (3). By contrast, maternal infection during the third trimester (late infection) leads to a greater risk of subclinical infection in newborns (up to $70 \%$ ) (3-5).

\section{Clinical manifestations}

Toxoplasmosis can be categorized, depending on the immune status of the patient, into 4 groups $(1,2)$.

1. Immunocompetent patients (children, adults, pregnant women) are predominantly asymptomatic. However, $10 \%-20 \%$ of those patients may develop cervical or occipital lymphadenopathy or a flu-like illness. Generally, symptoms are self-limited and resolve within weeks to months. Recent data have suggested an association between Toxoplasma gondii infection and various psychiatric or neurologic syndromes such as schizophrenia, Alzheimer syndrome, and even suicide $(7,8)$.

2. Immunodeficient patients. The reactivation of chronic situations may lead to life-threatening disease $(1,2)$. In these individuals, the Central Nervous System (CNS) is affected the most, and includes encephalitis, decrease of mental status, seizures, movement disorders and other psychiatric findings. Toxoplasmosis in immunodeficient patients can present as chorioretinitis, pneumonitis or involve multiple organs, finally leading to acute respiratory failure, myocarditis and hemodynamic abnormalities (1).

3. Ocular toxoplasmosis constitutes an important cause of chorioretinitis and may be the result of acquired or congenital infection $(1,2)$. Patients who have been infected by vertical transmission can be asymptomatic until the second and the third decade of life, when lesions develop in the eyes (1). Chorioretinitis in those individuals is more often bilateral (2).

4. In congenital toxoplasmosis, as has been said, the severity of clinical and ultrasonographic findings in congenitally infected fetuses is inversely related to the gestational age at the time of primary maternal infection. As a result, a first trimester maternal infection usually leads to more severe manifestations $(4,5,10)$. Prenatal ultrasonographic findings include intracranial calcifications, ventricular dilatation, hepatic enlargement, ascites, and increased placental thickness (9). In some cases, spontaneous abortion, prematurity, or stillbirth may occur $(1,2)$. In general, congenital toxoplasmosis is characterized by a wide spectrum of clinical manifestations, but in $70-90 \%$ of infected newborns it is subclinical, although involvement of CNS is a hallmark (1). Chorioretinitis, hydrocephalus and intracranial calcifications constitute the so-called classic triad of congenital toxoplasmosis (1). Other clinical manifestations include microcephaly, strabismus, blindness, epilepsy, mental retardation, petechiae due to thrombocytopenia, anemia, hepatosplenomegaly, jaundice, myocarditis, pneumonitis and respiratory distress (9).

\section{Differential Diagnosis and fetal diagnosis.}


At the beginning of pregnancy among the routine serological screening of the pregnant woman, the antitoxoplasma IgM and IgG antibodies are usually checked (3). In case both anti-toxoplasma (anti-toxo) IgM and IgG antibodies results are negative there is a follow up serological examination of them every 1,2 or 3 months. Since on follow up, they remain negative while fetal ultrasounds have no detectable abnormalities, no more actions are required. In case anti-toxo IgM antibodies are negative and IgG antibodies are positive, most probably the pregnant woman was infected in the distant past and before conception, which means no treatment is required (unless the pregnant woman is immunosuppressed). If anti-toxo IgM are positive, while anti-toxo IgG antibodies are negative, additional serological tests should be repeated in 1-3 weeks. In this case, since the after coming results are the same, the outcome is considered to be clinically insignificant, which means no treatment is required and a routine serological testing follow up is enough. However in case both anti-toxo IgM and IgG are positive after the follow up or even both of them are positive in the first test, an early infection of toxoplasma gondii is more likely to have occurred. In this case more serological tests should be performed in order to differentiate an early from a past infection and to find out whether the fetus has been infected too (11). To detect an early infection the main additional serological tests used are IgG avidity, anti-toxo IgA and IgE antibodies detection by the ELISA method.

Avidity is called the binding force of an antigen to its antibody. In the initial stages of antigenicity avidity is low, while after a few months it is increased by creating B cell antigens (12). The antigenic contact causes $\mathrm{B}$ cell maturation, while antibodies and antigens are binding stronger. In this way avidity will rise. High avidity can reject the possibility of acute infection. However, It seems that the combination of IgM and $\operatorname{IgE}$ anti-toxo antibody detection by ELISA is the most reliable method for acute infection diagnosis (13).

The main benefits of the anti toxo IgE antibody detection by ELISA, which make it an excellent supplementary method to diagnose acute toxoplasmosis, are its high specificity and positive predictive value with a sufficient sensitivity (14).

Finally, Toxoplasma gondii IgA antibody detection has been used in screening of pregnant women since they significantly increase the possibility of acute T. gondii infection in contrast to patients without them (15-17). However, some authors have found IgA either too insensitive or reactive for too long suggesting that, IgA testing is controversial and not a dependable marker for T. gondii acute infection detection (18).

For fetal toxoplasmosis infection, it is the amniotic fluid PCR analysis rather than the umbilical cord blood sample, the leading method for prenatal diagnosis. Despite the fact that this method provides fast results for parasite detection, it is also independent of a patient's immunological status and it has a broad spectrum of application in different biological samples. In addition, the protocol provides qualitative results (19).

Moreover, amniotic fluid PCR analysis should be done when primary infection is detected in pregnant women or even when serological tests are inconclusive whether an acute infection has occurred. Finally, PCR analysis is used in case of abnormal fetal ultrasound features have been detected, such as microcephaly, ascites, hepatosplenomegaly, hydrocephalus and severe IUGR (intrauterine growth restriction). Furthermore, in order to reduce the false negative results, amniotic fluid PCR should be performed 4 weeks after the suspected primary infection of the pregnant, but never before the 18th week of gestation. In case primary infection of the mother is confirmed while embryo infection is not, spiramycin has to be used for embryo protection, in order to avoid T.gondii transmission to the fetus across the placenta. Finally, when embryo infection is confirmed or highly suspected (in most cases by a positive amniotic fluid PCR analysis) pregnant women should receive pyrimethamine sulfadiazine and folic acid as a treatment (20).

Overall, this is one of the few cases reported based on the literature of a 19-year-old girl detected with toxoplasmosis during her pregnancy, managed successfully leading to a successful pregnancy. Early detection and medical intervention are necessary either to prevent embryo infection or treat embryos when infection is confirmed or suspected. Not only her young age but also her successful pregnancy as well, contributes to its interest for publication.

\section{Patient Perspective}


The patient was well informed since, the diagnosis of toxoplasmosis was confirmed, about the possible consequences. She was additionally well prepared about the possibility of terminating her pregnancy. Fortunately, with the proper follow up and interventions, throughout, her whole pregnancy she was finally pleased to make it through and deliver a healthy child.

\section{Informed consent}

A written informed consent was obtained from the patient for the publication of this case report.

\section{Acknowledgments}

The authors are grateful to all who provided assistance during the preparation of this manuscript.

Declaration of interest : The authors declare that they have no competing interest.

Study Funding : This research did not receive any specific grant from funding agencies in the public, commercial, or not-for-profit sectors.

Ethical committee: This study conforms the recognized standards according to the Declaration of Helsinki.

Author contributions :

Thomas Ntounis: Contributed to conception and design, drafted the manuscript, responsible for overall supervision.

Sofoklis Stavros: Contributed to conception and design, drafted the manuscript, responsible for overall supervision.

Antonios Koutras: Contributed to conception and design

Alexandros Katrachouras: Contributed to conception and design

Dimitrios Lentzaris: Contributed to conception and design

Aikaterini Domali: Responsible for overall supervision, drafted the manuscript

Kyriakos Konis: Responsible for overall supervision, drafted the manuscript

Peter Drakakis: Responsible for overall supervision, drafted the manuscript, overall revision.

All authors read and approved the final manuscript.

References

1. McAuley J. B. 2014. Congenital Toxoplasmosis. Journal of the Pediatric Infectious Diseases Society , 3 Suppl 1 (Suppl 1), S30-S35. https://doi.org/10.1093/jpids/piu077

2. Dubey, J. P., Miller, N. L., and Frenkel, J. K. 1970. The Toxoplasma gondii oocyst from cat feces. The Journal of experimental medicine , 132 (4), 636-662. https://doi.org/10.1084/jem.132.4.636

3. Montoya J. G. 2018. Systematic screening and treatment of toxoplasmosis during pregnancy: is the glass half full or half empty?. American journal of obstetrics and gynecology , 219 (4), 315-319. https://doi.org/10.1016/j.ajog.2018.08.001

4. Dunn, D., Wallon, M., Peyron, F., Petersen, E., Peckham, C., and Gilbert, R. 1999. Mother-to-child transmission of toxoplasmosis: risk estimates for clinical counselling. Lancet (London, England) , 353 (9167), 1829-1833. https://doi.org/10.1016/S0140-6736(98)08220-8

5. Daffos, F., Forestier, F., Capella-Pavlovsky, M., Thulliez, P., Aufrant, C., Valenti, D., and Cox, W. L. 1988. Prenatal management of 746 pregnancies at risk for congenital toxoplasmosis. The New England journal of medicine, 318(5), 271-275. https://doi.org/10.1056/NEJM198802043180502 
6. Kusbeci, O. Y., Miman, O., Yaman, M., Aktepe, O. C., and Yazar, S. 2011. Could Toxoplasma gondii have any role in Alzheimer disease?. Alzheimer disease and associated disorders, 25(1), 1-3. https://doi.org/10.1097/WAD.0b013e3181f73bc2

7. Arias, I., Sorlozano, A., Villegas, E., de Dios Luna, J., McKenney, K., Cervilla, J., Gutierrez, B., and Gutierrez, J. 2012. Infectious agents associated with schizophrenia: a meta-analysis. Schizophrenia research , 136 (1-3), 128-136. https://doi.org/10.1016/j.schres.2011.10.026

Gay-Andrieu, F., Marty, P., Pialat, J., Sournies, G., Drier de Laforte, T. and Peyron, F. 2003. Fetal toxoplasmosis and negative amniocentesis: necessity of an ultrasound follow-up. Prenatal diagnosis , 23 (7), 558-560. https://doi.org/10.1002/pd.632

Swisher, C. N., Boyer, K. and McLeod, R. 1994. Congenital toxoplasmosis. The Toxoplasmosis Study Group. Seminars in pediatric neurology, 1(1), 4-25.

10. Kieffer, F. and Wallon, M. 2013. Congenital toxoplasmosis. Handbook of clinical neurology, 112, 10991101. https://doi.org/10.1016/B978-0-444-52910-7.00028-3

11. Olariu, T. R., Blackburn, B. G., Press, C., Talucod, J., Remington, J. S. and Montoya, J. G. 2019. Role of Toxoplasma IgA as Part of a Reference Panel for the Diagnosis of Acute Toxoplasmosis during Pregnancy. Journal of clinical microbiology, 57(2), e01357-18. https://doi.org/10.1128/JCM.01357-18

12. Rahbari, A. H., Keshavarz, H., Shojaee, S., Mohebali, M. and Rezaeian, M. 2012. IgG avidity ELISA test for diagnosis of acute toxoplasmosis in humans. The Korean journal of parasitology, 50(2), 99-102. https://doi.org/10.3347/kjp.2012.50.2.99

13.Sharifi, K., Hosseini Farash, B. R., Tara, F., Khaledi, A., Sharifi, K. and Shamsian, S. 2019. Diagnosis of Acute Toxoplasmosis by IgG and IgM Antibodies and IgG Avidity in Pregnant Women from Mashhad, Eastern Iran. Iranian journal of parasitology , 14 (4), 639-645.

14. Kodym, P., Machala, L., Rohácová, H., Sirocká, B. and Malý, M. 2007. Evaluation of a commercial IgE ELISA in comparison with IgA and IgM ELISAs, IgG avidity assay and complement fixation for the diagnosis of acute toxoplasmosis. Clinical microbiology and infection : the official publication of the European Society of Clinical Microbiology and Infectious Diseases , 13 (1), 40-47. https://doi.org/10.1111/j.14690691.2006.01564.x

15. Olariu, T. R., Blackburn, B. G., Press, C., Talucod, J., Remington, J. S. and Montoya, J. G. 2019. Role of Toxoplasma IgA as Part of a Reference Panel for the Diagnosis of Acute Toxoplasmosis during Pregnancy. Journal of clinical microbiology, 57 (2), e01357-18. https://doi.org/10.1128/JCM.01357-18

16.Kodym, P., Machala, L., Rohácová, H., Sirocká, B. and Malý, M. 2007. Evaluation of a commercial IgE ELISA in comparison with IgA and IgM ELISAs, IgG avidity assay and complement fixation for the diagnosis of acute toxoplasmosis. Clinical microbiology and infection : the official publication of the European Society of Clinical Microbiology and Infectious Diseases , 13 (1), 40-47. https://doi.org/10.1111/j.14690691.2006.01564.x

17. Olariu, T. R., Blackburn, B. G., Press, C., Talucod, J., Remington, J. S. and Montoya, J. G. 2019. Role of Toxoplasma IgA as Part of a Reference Panel for the Diagnosis of Acute Toxoplasmosis during Pregnancy. Journal of clinical microbiology, 57(2), e01357-18. https://doi.org/10.1128/JCM.01357-18

18. Nascimento, F. S., Suzuki, L. A. and Rossi, C. L. 2008. Assessment of the value of detecting specific IgA antibodies for the diagnosis of a recently acquired primary Toxoplasma infection. Prenatal diagnosis, 28(8), 749-752. https://doi.org/10.1002/pd.2052

19. Breeze A. C. 2007. Infectious diseases of the fetus and newborn infant, 6th edn. Archives of Disease in Childhood. Fetal and Neonatal Edition, 92 (2), F156. https://doi.org/10.1136/adc.2006.102566 
20. Paquet, C., Yudin, M. H. and Society of Obstetricians and Gynaecologists of Canada 2013. Toxoplasmosis in pregnancy: prevention, screening, and treatment. Journal of obstetrics and gynaecology Canada: JOGC = Journal d'obstetrique et gynecologie du Canada : JOGC , 35 (1), 78-81. https://doi.org/10.1016/s17012163(15)31053-7

Figure 1

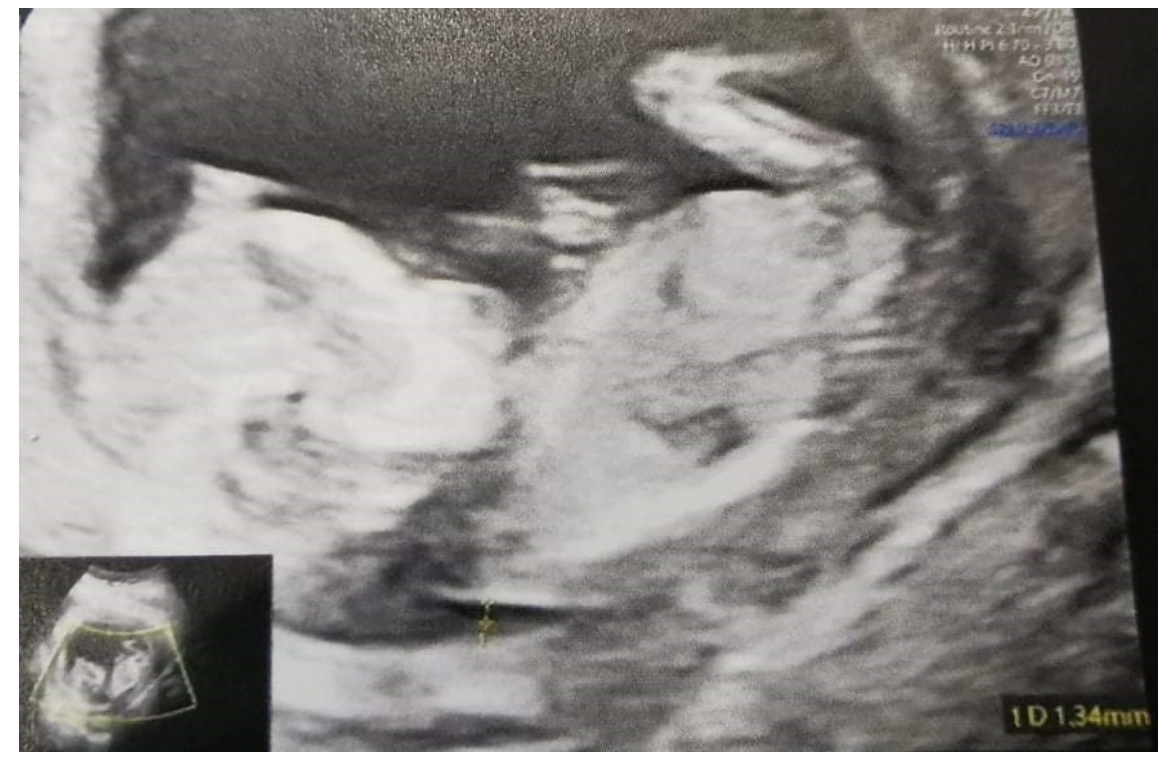

Fetal nuchal translucency ultrasonography $12^{\text {th }}$ week of gestation.

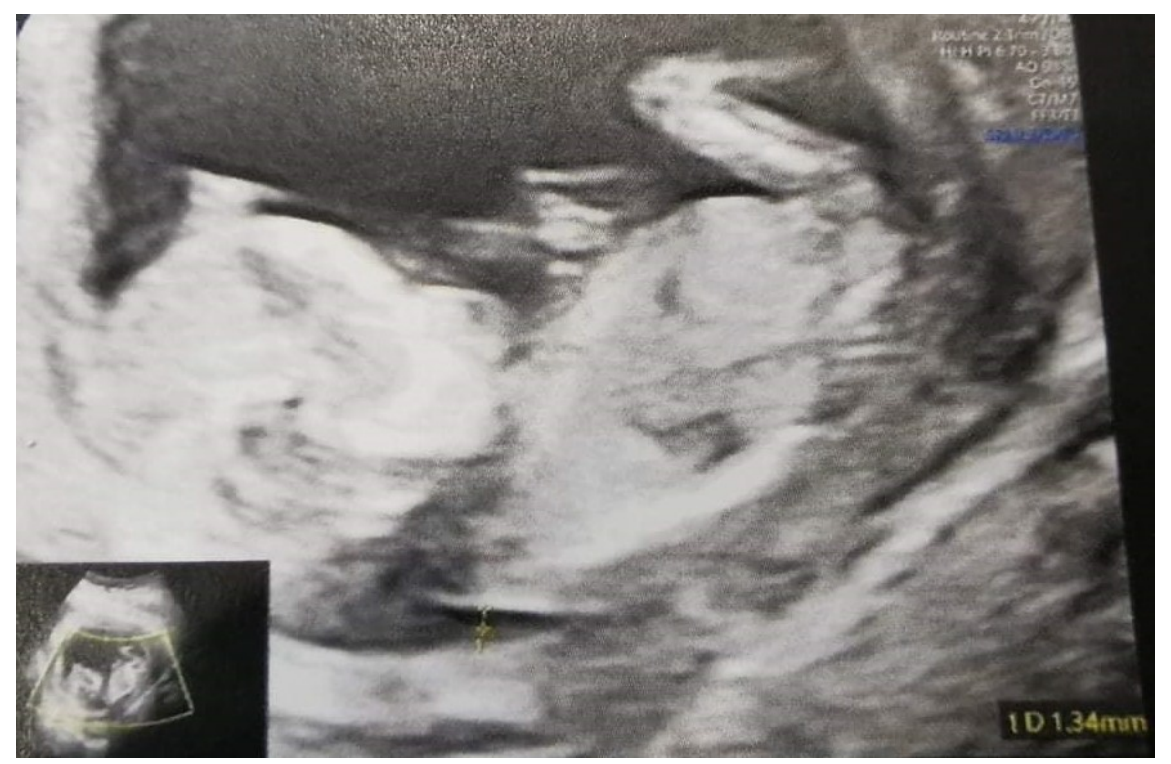

